\title{
Intense arousal of novel male mice in proximity to previously inseminated females: Inactivation of males via chlorpromazine does not diminish the capacity to disrupt pregnancy
}

\author{
DENYS DECATANZARO, CAMERON MUIR, EMILY SPIRONELLO, \\ TAMARA BINGER, and JEFF THOMAS \\ McMaster University, Hamilton, Ontario, Canada
}

\begin{abstract}
In the course of investigating early pregnancy disruptions by novel males, intense behavioral interactions and chemical emissions were observed among males housed in proximity to inseminated females. These interactions were evident despite wire-mesh separations between males and females. Experiments were designed to examine whether such arousal plays a role in pregnancy disruption. During the first 5 days of pregnancy, inseminated CF-1 strain female mice were either left undisturbed or exposed to novel outbred males. Physical separations between males did not diminish the frequency of pregnancy disruption. Subsequently, we investigated whether heavy sedation of novel males via chronic chlorpromazine administration would diminish the capacity to disrupt pregnancy. Pregnancy was disrupted to the same degree in females exposed to drug-treated males and those exposed to alert competing males given vehicle injections. These data show an unprecedented degree of arousal and pheromonal emission among competing males but suggest that these factors are not critical for the capacity to disrupt pregnancy.
\end{abstract}

Novel males can disrupt intrauterine implantation of fertilized ova in various rodents (see, e.g., Bruce, 1960a, 1960b; deCatanzaro, Zacharias, \& Muir, 1996; Heske \& Nelson, 1984; Storey, 1996), a phenomenon known as the Bruce effect. Diverse stressors also can have similar effects on implantation in many mammals (deCatanzaro \& MacNiven, 1992).

Several studies implicate pheromones of novel males in the Bruce effect. Some studies show pregnancy disruptions when females are merely exposed to excretions of males (e.g., Brennan, Schellinck, \& Keverne, 1999; Bruce, 1960b; deCatanzaro, Muir, Sullivan, \& Boissy, 1999; Dominic, 1965, 1966; Marchlewska-Koj, 1981; Parkes \& Bruce, 1962). Generally, it is much more difficult to produce an effect by excretions alone than by more direct exposure to novel males, and there are some methodological concerns (deCatanzaro et al., 1999). That pheromones contribute to the Bruce effect is also suggested by evidence that it can be prevented when females' chemosensation has been surgically disrupted (Bellringer, Pratt, \& Keverne, 1980; Bruce \& Parrott, 1960; Lloyd-Thomas \& Keverne, 1982; Rajendren \& Dominic, 1986).

This research was supported by grants from the Natural Sciences and Engineering Research Council of Canada to D. deCatanzaro. We appreciate the assistance of Kammie Bribriesco and Rose Zacharias. Correspondence concerning this article should be addressed to $D$. deCatanzaro, Department of Psychology, McMaster University, Hamilton, ON, L8S 4KI Canada (e-mail: decatanz@mcmaster.ca).
Behavioral factors can also contribute to the Bruce effect and may summate or act convergently with pheromonal factors. When novel males are housed directly with inseminated females, there are typically intense behavioral interactions between the males and the females. Novel males, unlike the sires of the pregnancies, are agitated and prone to approach and sexually mount previously inseminated females (deCatanzaro \& Storey, 1989; deCatanzaro et al., 1996). In many instances, the female is reinseminated by the novel male, as is evidenced by mating behavior, gestation length, and the resemblance of phenotype of the litter to that of the novel male. Some studies suggest that sexual interactions with the novel male, especially intromissions, could contribute to such pregnancy disruptions (deCatanzaro \& Storey, 1989; deCatanzaro et al., 1995).

When novel males are separated from females by a wire-mesh grid, pregnancy disruption is observed if the males are housed directly above the females, but not when they are housed below the females (deCatanzaro et al., 1996). Further spatial separation of novel males from previously inseminated females - for example, by doubling the grid separation-tends to diminish the likelihood of pregnancy disruption. The males of a strain distinct from that of the females produce a more reliable effect than do those of the same strain as the females (Marsden \& Bronson, 1965; Spironello \& deCatanzaro, 1999). Although males cannot mate with and reinseminate females in this context, they are difficult for humans to handle and appear to be very agitated. Thus, even where 
direct sexual activity is prevented, it is conceivable that there remains a behavioral element in the Bruce effect.

The initial experiments reported here were designed in accordance with the logic of Parkes and Bruce (1962), that the excretions of multiple novel males are more effective in disrupting pregnancy than are those of just one novel male. This was intended to provide a strong and robust paradigm of the Bruce effect, to set the stage for studies of physiological contributions to this effect. The designs were based on the understanding that male mice can coexist in groups in good health for weeks, with only transitory agonistic behavior that subsides after initial pairing (deCatanzaro \& Ngan, 1983). However, intense competition among the males, with emission of toxic pheromones, led to modification of the protocol. Subsequent to these unanticipated consequences, experiments were designed to examine whether physical separation and sedation of males might prevent their capacity to disrupt pregnancy and, thus, to determine whether such arousal of males is necessary for pregnancy disruption. Sedation of novel males was accomplished via chronic administration of chlorpromazine, a catecholaminergic receptor blocker that produces general inactivity and that has been found to disrupt male sexual activity (Soulairac \& Soulairac, 1975).

\section{METHOD}

\section{Subjects}

CF-1 strain mice (Mus musculus) were obtained from Charles River Breeding Farms of Canada, La Prairie, Quebec, or were bred in our laboratory from such stock. Heterogeneous strain (HS) mice were bred in this laboratory in the manner described previously (deCatanzaro et al., 1999). Housing involved standard polypropylene cages measuring $28 \times 16 \times 11$ (height) $\mathrm{cm}$ with straight-wire tops allowing continuous access to food and water, except where otherwise specified. The colony room was maintained under a reversed 14:10-h light:dark cycle at $21^{\circ} \mathrm{C}$.

\section{Insemination}

CF-1 female subjects, between 75 and 100 days of age, were each housed directly with 1 sexually experienced CF-1 male in a standard cage. Their hindquarters were inspected three times daily for the presence of a sperm plug. Females with sperm plugs were identified as subjects, and the day of detection was designated as Day 0 of pregnancy. Each female remained housed with the inseminating male until the morning after detection of a sperm plug, about the start of the dark phase of the lighting cycle. The female was then removed from the male, housed individually in a clean cage with fresh bedding, and assigned to one of the experimental conditions, counterbalanced across age and date of insemination.

\section{Apparatus}

A double-decker apparatus, described in detail previously (deCatanzaro et al., 1996), was used for exposing inseminated female CF-1 subjects to novel HS male mice. Briefly, this apparatus was constructed from clear Plexiglas, measuring $30 \times 21 \times 27 \mathrm{~cm}$, divided into upper and lower compartments (each measuring $30 \times$ $21 \times 13 \mathrm{~cm}$ ) by a wire-mesh grid (squares of $0.5 \mathrm{~cm}^{2}$ ). The lower compartment was filled with approximately $0.5 \mathrm{~L}$ of clean bedding and had independent food and water supplies. The upper compartment was covered with a standard straight-wire mouse cage lid providing continuous access to food and water.

\section{Novel Males in the Experimental Apparatus}

Inseminated female subjects were randomly assigned to conditions involving housing below 2 HS males or housing below no males (control), for a 120-h period commencing at the start of the dark phase of the light cycle on Day 1 of pregnancy. Initially, samples were conducted with 2 HS males housed directly together in the upper compartment of the experimental apparatus. Then, samples were run in which the upper compartment of each apparatus was divided into two portions by a wire-mesh grid, with 1 male housed on each side of this partition. Following this, samples were run with a double opaque Plexiglas barrier being used to separate the males into two compartments.

\section{Exposure of Females to Chlorpromazine- and Vehicle-Treated Males}

Inseminated females were randomly assigned to three conditions that involved a $96-\mathrm{h}$ period in the lower portion of the experimental apparatus, commencing at the start of the dark phase of the light cycle on Day 2 after insemination. The period of exposure was reduced by 1 day from previous experiments, so that full sedation of novel males could be more easily sustained. In the control condition, each female was housed in the lower portion, with no stimulus animals housed above. For the other two conditions, females were each housed in the lower portion of the apparatus below 2 novel males. In the chlorpromazine and vehicle conditions, HS males were housed in pairs, as was described above, in the upper portion of the double-decker apparatus, separated by double opaque Plexiglas barriers.

\section{Chronic Chlorpromazine Administration}

Preliminary trials were carried out to determine the dosage and timing of chlorpromazine that would optimize sedation. A dose of $0.36 \mathrm{mg}$ in $0.05 \mathrm{cc}$ saline/animal administered intraperitoneally initially produced complete sedation for more than $12 \mathrm{~h}$, with minimal movement and unresponsiveness to human touch. However, animals showed some tolerance with repeated injections of this dosage, so it was determined that the dosage should be stepped up and the intervals between injections shortened, to sustain full sedation. Each animal received seven injections during a 4-day period of exposure to females. For the condition involving chlorpromazinetreated males, an initial injection of $0.36 \mathrm{mg} / \mathrm{animal}$ was administered upon introduction to females at $11.5 \mathrm{~h}$ after commencement of the dark phase of the animals' light cycle. Subsequently, at 12-h intervals, each male received second, third, and fourth doses of $0.36 \mathrm{mg}$. Then, at 8-h intervals, fifth, sixth, and seventh doses, each of $0.56 \mathrm{mg}$, also in $0.05 \mathrm{cc}$ saline, were administered. Fresh solutions of chlorpromazine in saline were prepared just prior to each injection. For the (control) condition involving vehicle treatment, each male received an injection of $0.05 \mathrm{cc}$ saline according to the same time schedule as that in the drug-treated animals.

\section{Dependent Measures}

After the experimental manipulations ended, subjects were not disturbed until 18 days after sperm plug detection, at which point they were observed twice daily for potential parturition until Day 23 after sperm plug detection. The maximal length of normal gestation in this strain of mice in this laboratory is 22 days. Pregnancy outcome was measured through counts of the number of pups born.

\section{RESUI TS}

Initially, when there was no partition in the upper compartment between the 2 males, unanticipated behavioral dynamics emerged between the males. In 10 of the 19 cases in which novel males were housed above inseminated females, 1 male of the pair died. This result was not 
anticipated or intended. In 5 cases, this occurred on Day 2 after introduction into the apparatus, 2 other cases occurred on Day 3, and in 3 other cases, males apparently about to succumb to this syndrome were removed from the apparatus prior to death on Day 4, as will be described below. In all 10 cases, the remaining male was in apparent good health with few wounds. Each deceased male had bite marks that appeared insufficient to account for mortality, but its fur and skin were completely covered in an oily and odorous substance apparently excreted by the victor (since it was also present, but in much smaller amounts, on the latter's ventral surface, proximate to the penis). Deceased males were removed as soon as detected but were not replaced. In 3 cases, on Day 4 of exposure to females in the apparatus, 1 male in the upper compartment was observed to be sluggish and covered completely in the oily emission and was removed from the apparatus and housed individually. This did not prevent death, however, which ensued from 10 to $18 \mathrm{~h}$ later in each case. In the interests of animal care, these procedures were subsequently modified by the introduction of barriers between the males.

Where a wire-mesh grid was introduced between males, agonistic behavior remained despite the barrier. Males in six cases were observed to interact through the wire-mesh grid, hovering by it and attempting attacks. In two cases, there was mortality of one of the males, on Day 2 after introduction into the apparatus, with the male covered in the same oily emission as described above. These males were not replaced. Where males were separated by doubled opaque plastic, no behavioral interactions occurred between males, and all males remained in good health. We observed that males continued to urinate in the vicinity of the barrier and that they remained exceptionally difficult to handle.

Table 1 provides data from initial studies in which 2 HS males were housed above each female with varied forms of separation between the males. The number of pups born is presented in two ways: (1) mean pups/female when considering all the females (with zero values for nonparturient females), and (2) mean pups/female when considering only the subset of females that were parturient. With no partition in the upper compartment between the 2 males, 13 of 14 control females were parturient, whereas 7 of 19 male-exposed females were parturient. A test of association, relating the two conditions to the presence or absence of a litter, showed significance $\left[\chi^{2}(1)=\right.$ $10.59, p<.005]$. When the number of pups born to all the females was considered (with a value of zero for each nonparturient female), an analysis of variance (ANOVA) was significant $[F(1,31)=13.80, p=.001]$. A correlation of male mortality and birth of a litter (each coded as 1 if it occurred and as 0 if not) among the 19 experimental cases was negligible $[r=-.15, t(17)=0.623, p$ $=.55]$. In samples in which novel males were separated by wire mesh, 11 of 12 control females and 3 of 12 maleexposed females were parturient $\left[\chi^{2}(1)=10.97, p<\right.$ $.001]$. An ANOVA on the number of pups born was significant $[F(1,22)=19.73, p<.001]$. Where males were separated by doubled opaque plastic, control females were parturient in 18 of 20 cases, whereas 5 of 20 maleexposed females were parturient. A test of association, relating the two conditions to the presence or absence of a litter, showed significance $\left[\chi^{2}(1)=17.29, p<.001\right]$. An ANOVA on the number of pups born was also significant $[F(1,38)=26.22, p<.001]$.

Chlorpromazine administration resulted in substantial motor retardation; all males remained immobile in response to human injections and handling throughout exposure to previously inseminated females. All the males survived the period of exposure to females and recovered normal activity following cessation of drug treatment. Table 2 presents pregnancy outcome in females after exposure to no males (control), vehicle-treated males, or chlorpromazine-treated males. All 15 of 15 control females were parturient, whereas 6 of 15 females exposed to vehicle-treated and 6 of 15 females exposed to chlorpromazine-treated males were parturient. A test of association, relating all three conditions to the presence or absence of a litter, showed significance $\left[\chi^{2}(2)=12.26\right.$, $p<.005]$. When each pair of conditions was compared through a test of association, there were significant differences between the control and the vehicle conditions $\left[\chi^{2}(1)=10.77, p<.005\right]$ and between the control and chlorpromazine conditions $\left[\chi^{2}(1)=10.77, p<.005\right]$. The number of pups born to all the females (with a value of zero for each nonparturient female) was found to be significantly different by an ANOVA $[F(2,42)=8.91, p<$

Table 1

Mean $( \pm S E$ ) Number of Pups Born and Number Parturient in Inseminated CF-1 Females Exposed During Days 1-5 of Pregnancy to No Male (Control) or to Two Heterogeneous Strain Males With Varied Forms of Separation Between the Males

\begin{tabular}{lccc}
\hline \multicolumn{1}{c}{ Form of Separation } & Number of Pups/Female & Pups/Litter & \% Parturient \\
\hline Males without any partition & & & \\
$\quad$ Control & $10.79 \pm 1.10$ & $11.62 \pm 0.78$ & 92.9 \\
$\quad$ Male exposed & $4.11 \pm 1.31$ & $11.14 \pm 0.96$ & 36.8 \\
Males separated by wire mesh & & & \\
$\quad$ Control & $11.00 \pm 1.22$ & $12.00 \pm 0.76$ & 91.7 \\
$\quad$ Male exposed & $2.67 \pm 1.43$ & $10.67 \pm 1.45$ & 25.0 \\
Males separated by double opaque barrier & & & \\
$\quad$ Control & $10.05 \pm 0.95$ & $11.17 \pm 0.62$ & 90.0 \\
$\quad$ Male exposed & $2.65 \pm 1.09$ & $10.60 \pm 1.21$ & 25.0 \\
\hline
\end{tabular}


Table 2

Mean $( \pm S E$ ) Number of Pups Born and Number Parturient in Inseminated CF-1 Females Indirectly Exposed During Days 2-5 of Pregnancy to No Male (Control), Vehicle-Treated Males, or Chlorpromazine-Treated Males

\begin{tabular}{lccc}
\hline \multicolumn{1}{c}{ Males } & Number of Pups/Female & Pups/Litter & $\%$ Parturient \\
\hline Control & $12.13 \pm 0.69$ & $12.13 \pm 0.69$ & 100.0 \\
Vehicle-treated & $4.87 \pm 1.67$ & $12.17 \pm 1.35$ & 40.0 \\
Chlorpromazine-treated & $4.87 \pm 1.63$ & $12.17 \pm 2.23$ & 40.0 \\
\hline
\end{tabular}

$.001]$. Multiple comparisons (Newman-Keuls, $p<.01$ ) indicated that the number of pups in the control condition differed from that in the vehicle condition and that the control condition differed from the chlorpromazine condition.

\section{DISCUSSION}

The initial experiments were designed to examine the influence of 2 novel males on pregnancy in nearby inseminated females. Unexpectedly, intense behavioral interactions were observed between the males in these pairs. Under these circumstances, males became agitated and difficult for humans to handle, hovered above females, excreted milky-white and oily odorous substances, and became exceptionally aggressive if other males were in their proximity. Again unexpectedly, these interactions were not eliminated by separation of the males by a wiremesh grid. Behavioral interactions between males were prevented only when males could not physically contact one another because of an opaque plastic barrier, but males continued to urinate on the barrier. These experiments suggest that exceptionally intense behavioral arousal may be produced in males housed in proximity to inseminated females but without full access to these females.

In order to examine the possibility that behavioral arousal contributes to the Bruce effect in this context, a subset of males was chronically given a major tranquilizer, chlorpromazine. Females exposed to such males showed pregnancy disruption with a frequency similar to that found when males were active. These data suggest that behavioral arousal of novel males may not be necessary for pregnancy disruption to occur. The regimen of chronic chlorpromazine administration in this study induced sustained inactivity and motor retardation in all the males on this regimen. This regimen involves the maximal dosage possible without inducing hypothermia; therefore, it could not be argued that the dose was insufficient to impede males' capacity to disrupt pregnancy.

Storey (1996) observed that novel male meadow voles were less likely to disrupt the pregnancy of previously inseminated female meadow voles (Microtus pennsylvanicus) when administered clonidine, an $\alpha 2$-adrenergic receptor agonist that decreases general activity, than when given saline. Clonidine administration disrupts sexual activity in males (Clark, 1991). Storey's pregnancy disruption paradigm, unlike that of the present study, in- volved direct sexual contact between the novel males and the previously inseminated females, which may be a necessary condition for pregnancy disruption in meadow voles. In mice (Mus musculus), it is possible to produce pregnancy disruptions either with direct contact, including sexual activity, or with reduced contact that prevents sexual activity, as in the present study, but the qualities of the effect in the two conditions differ (deCatanzaro et al., 1996). Thus, differences in species, in paradigms, and in mechanisms of drug action may account for the apparent discrepancy between the results of the present study and those of Storey (1996).

The present study reports that exceptionally intense agonistic behavior was engendered by pairing males proximate to a previously inseminated female without sexual access. Although intermale competition is well known in laboratory mice (see, e.g., Brain \& Benton, 1983; deCatanzaro, 1999) and female exposure has been shown to stimulate agonistic behavior (deCatanzaro, 1981), the qualities of competition observed here, involving enurination targeted at the opponent, may be unprecedented. A comparable effect has been reported for mature male guinea pigs paired in an enclosure in the presence of an unfamiliar female (Sachser \& Lick, 1989; Sachser, Lick, \& Stanzel, 1994). When opponents were chronically kept together continuously for a period of 8 days, usually one of the males, but not the other, fell into a comatose state and died 5-8 days after the confrontation. As in the present study, wounding was insufficient to account for this effect. We suspect toxification by the oily emissions that were observed on subordinated mice; these emissions were, however, not reported for the guinea pig and, to the best of our knowledge, have never previously been reported for mice. In any event, the fact that the Bruce effect occurs despite physical separation of males and despite behavioral inactivation of males by chlorpromazine injections suggests that arousal state and competition between males is not relevant to the Bruce effect.

\section{REFERENCES}

Bellringer, J. F., Pratt, H. P. M., \& Keverne, E. B. (1980). Involvement of the vomeronasal organ and prolactin in pheromonal induction of delayed implantation in mice. Journal of Reproduction \& Fertility, 50, 223-228.

Brain, P. F., \& BEnTON, D. (1983). Housing, hormones, and aggressive behavior. In B. B. Svare (Ed.), Hormones and aggressive behavior (pp. 351-372). New York: Plenum.

Brennan, P. A., Schellinck, H. M., \& Keverne, E. B. (1999). Pat- 
terns of expression of the immediate-early gene egr- 1 in the accessory olfactory bulb of female mice exposed to pheromonal constituents of male urine. Neuroscience, 90, 1463-1470.

Bruce, H. M. (1960a). A block to pregnancy in mice caused by the proximity of strange males. Journal of Reproduction \& Fertility, 1 , 96-103.

BRUCE, H. M. (1960b). Further observations of the pregnancy block in mice caused by the proximity of strange males. Journal of Reproduction \& Fertility, 1, 311-312.

Bruce, H. M., \& Parrott, D. M. V. (1960). Role of olfactory sense in pregnancy block by strange males. Science, 131, 1526.

CLARK, J. (1991). Suppression of copulatory behavior in male rats following central administration of clonidine. Neuropharmacology, $\mathbf{3 0}$, 373-382.

DeCaTANZaro, D. (1981). Facilitation of intermale aggression in mice through exposure to receptive females. Journal of Comparative \& Physiological Psychology, 95, 638-645.

DeCATANzaro, D. (1999). Motivation and emotion: Evolutionary, physiological, developmental, and social perspectives. Upper Saddle River, NJ: Prentice Hall.

deCatanzaro, D., \& MacNiven, E. (1992). Psychogenic pregnancy disruptions in mammals. Neuroscience \& Biobehavioral Reviews, 16, 43-53.

deCatanzaro, D., Muir, C., Sullivan, C., \& Boissy, A. (1999). Pheromones and novel-male-induced pregnancy disruption in mice: Exposure to conspecifics is necessary for urine alone to induce an effect. Physiology \& Behavior, 66, 153-157.

deCatanzaro, D., \& NGan, E. T. (1983). Dominance in intermale encounters and subsequent sexual success in mice. Journal of Comparative Psychology, 97, 269-278.

deCatanzaro, D., \& Storey, A. (1989). Partial mediation of strangemale-induced pregnancy blocks by sexual activity in mice (Mus musculus). Journal of Comparative Psychology, 103, 381-388.

deCatanzaro, D., Wyngaarden, P., Griffiths, J., Ham, M., HanCOX, J., \& BraIN, D. (1995). Interactions of contact, odor cues, and androgens in strange-male-induced early pregnancy disruptions in mice (Mus musculus). Journal of Comparative Psychology, 109, 115 122.

deCatanzaro, D., Zacharias, R., \& MuiR, C. (1996). Distuption of early pregnancy by direct and indirect exposure to novel males in mice: Comparison of influences of preputialectomized and intact males. Journal of Reproduction \& Fertility, 106, 269-274.
DominIC, C. J. (1965). The origin of pheromones causing pregnancy block in mice. Journal of Reproduction \& Fertility, 10, 469-472.

Dominic, C. J. (1966). Observations on the reproductive pheromones of mice: I. Source. Journal of Reproduction \& Fertility, 11, 407-414.

Heske, E. J., \& Nelson, R. J. (1984). Pregnancy interruption in Microtus ochrogaster: Laboratory artifact or field phenomenon. Biology of Reproduction, 31, 97-103.

Lloyd-Thomas, A., \& Keverne, E. B. (1982). Role of the brain and accessory olfactory system in the block to pregnancy in mice. Neuroscience, 7, 907-913.

MarChlewSKa-KoJ, A. (1981). Pregnancy block elicited by male urinary peptides in mice. Journal of Reproduction \& Fertility, 61, 221224.

MARSDEN, H. M., \& BRonson, F. H. (1965). Strange male block to pregnancy: Its absence in inbred mouse strains. Nature, 207, 878 .

Parkes, A. S., \& Bruce, H. M. (1962). Pregnancy block in female mice placed in boxes soiled by males. Journal of Reproduction \& Fertility, 4, 303-308.

RaJendRen, G., \& Dominic, C. J. (1986). Effect of bilateral transection of the lateral olfactory tract on the male-induced implantation failure. Physiology \& Behavior, 36, 587-590.

SACHSER, N., \& Lick, C. (1989). Social stress in guinea pigs. Physiology \& Behavior, 46, 137-144.

SACHSER, N., Lick, C., \& StAnzel, K. (1994). The environment, hormones, and aggressive behavior: A 5 -year-study in guinea pigs. Psychoneuroendocrinology, 19, 697-707.

Soulairac. M.-L., \& Soulairac, A. (1975). Monoaminergic and cholinergic control of sexual behavior in the male rat. In M. Sandler \& G. L. Gessa (Eds.), Sexual behavior: Pharmacology and biochemistry (pp. 99-116). New York: Raven.

Spironello, E., \& deCatanzaro, D. (1999). Sexual satiety diminishes the capacity of novel males to disrupt early pregnancy in inseminated female mice (Mus musculus). Journal of Comparative Psychology, $113,1-5$.

STOREY, A. (1996). Behavioral interactions increase pregnancy blocking by unfamiliar male meadow voles. Physiology \& Behavior, 60, 1093-1098.

(Manuscript received June 21, 1999; revision accepted for publication September $7,1999$.

\section{Forthcoming Articles}

The forthcoming articles list for Psychobiology has not been included in the present issue. It can be found at www.psychonomic.org. 